\title{
The Revolutionary Calendar - An II
}

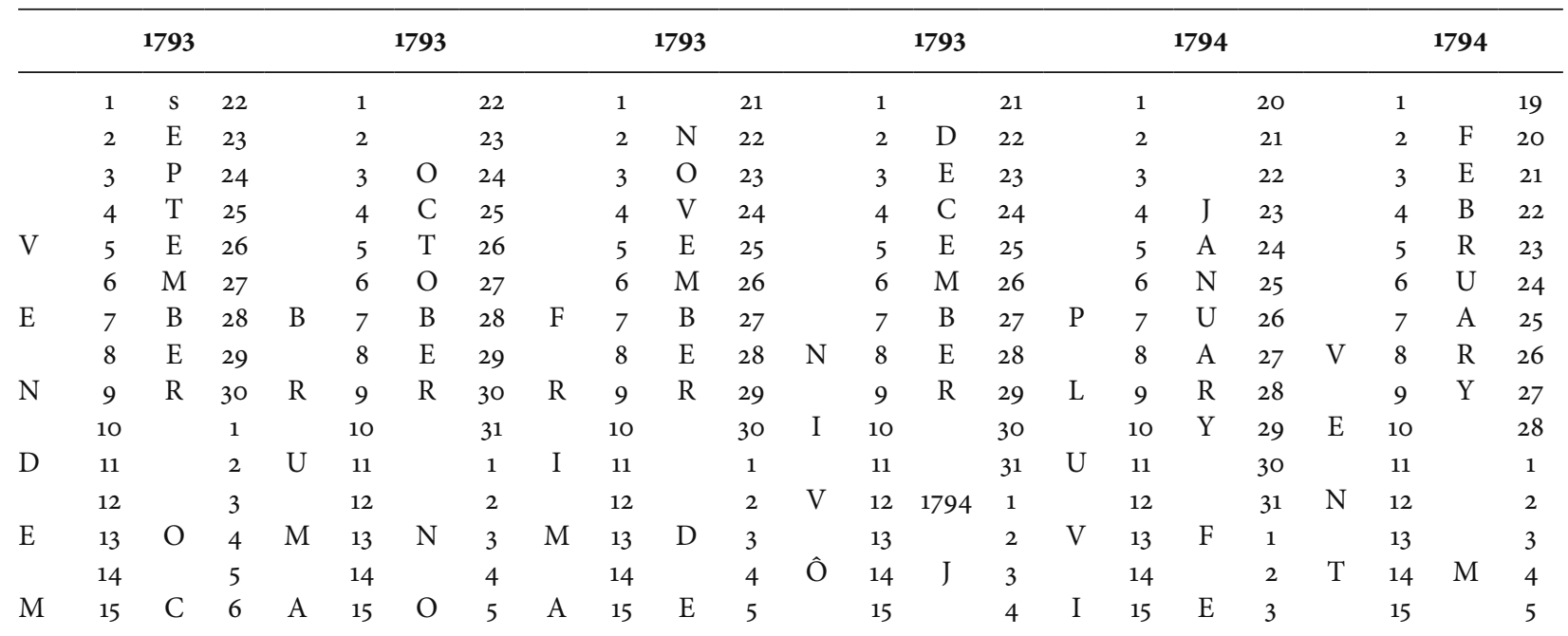




\begin{tabular}{cccccccccccccccccccccccccc} 
& 16 & & 7 & & 16 & & 6 & & 16 & & 6 & $\mathrm{~S}$ & 16 & $\mathrm{~A}$ & 5 & & 16 & & 4 & $\mathrm{O}$ & 16 & $\mathrm{~A}$ & 6 \\
$\mathrm{~A}$ & 17 & $\mathrm{~T}$ & 8 & $\mathrm{I}$ & 17 & $\mathrm{~V}$ & 7 & $\mathrm{I}$ & 17 & $\mathrm{C}$ & 7 & & 17 & & 6 & $\mathrm{O}$ & 17 & $\mathrm{~B}$ & 5 & & 17 & & 7 \\
& 18 & & 9 & & 18 & & 8 & & 18 & & 8 & $\mathrm{E}$ & 18 & $\mathrm{~N}$ & 7 & & 18 & & 6 & $\mathrm{~S}$ & 18 & $\mathrm{R}$ & 8 \\
$\mathrm{I}$ & 19 & $\mathrm{O}$ & 10 & $\mathrm{R}$ & 19 & $\mathrm{E}$ & 9 & $\mathrm{R}$ & 19 & $\mathrm{E}$ & 9 & & 19 & & 8 & $\mathrm{~S}$ & 19 & $\mathrm{R}$ & 7 & & 19 & & 9 \\
& 20 & & 11 & & 20 & & 10 & & 20 & & 10 & & 20 & $\mathrm{U}$ & 9 & & $2 \mathrm{O}$ & & 8 & $\mathrm{E}$ & 20 & $\mathrm{C}$ & 10 \\
$\mathrm{R}$ & 21 & $\mathrm{~B}$ & 12 & $\mathrm{E}$ & 21 & $\mathrm{M}$ & 11 & $\mathrm{E}$ & 21 & $\mathrm{M}$ & 11 & & 21 & & 10 & $\mathrm{E}$ & 21 & $\mathrm{U}$ & 9 & & 21 & & 11 \\
& 22 & & 13 & & 22 & & 12 & & 22 & & 12 & & 22 & $\mathrm{~A}$ & 11 & & 22 & & 10 & & 22 & $\mathrm{H}$ & 12 \\
$\mathrm{E}$ & 23 & $\mathrm{E}$ & 14 & & 23 & $\mathrm{~B}$ & 13 & & 23 & $\mathrm{~B}$ & 13 & & 23 & & 12 & & 23 & $\mathrm{~A}$ & 11 & & 23 & 13 \\
& 24 & & 15 & & 24 & & 14 & & 24 & & 14 & & 24 & $\mathrm{R}$ & 13 & & 24 & & 12 & & 24 & 14 \\
& 25 & $\mathrm{R}$ & 16 & & 25 & $\mathrm{E}$ & 15 & & 25 & $\mathrm{E}$ & 15 & & 25 & & 14 & & 25 & $\mathrm{R}$ & 13 & & 25 & 15 \\
& 26 & & 17 & & 26 & & 16 & & 26 & & 16 & & 26 & $\mathrm{Y}$ & 15 & & 26 & & 14 & & 26 & 16 \\
& 27 & & 18 & & 27 & $\mathrm{R}$ & 17 & & 27 & $\mathrm{R}$ & 17 & & 27 & & 16 & & 27 & $\mathrm{Y}$ & 15 & & 27 & & 17 \\
& 28 & & 19 & & 28 & & 18 & & 28 & & 18 & & 28 & & 17 & & 28 & & 16 & & 28 & 18 \\
& 29 & 20 & & 29 & & 19 & & 29 & & 19 & & 29 & & 18 & & 29 & & 17 & & 29 & 19 \\
& 30 & 21 & & 30 & & 20 & & 30 & & 20 & & 30 & & 19 & & 30 & & 18 & & 30 & 20 \\
\hline
\end{tabular}




\section{The Revolutionary Calendar - An II}

\begin{tabular}{|c|c|c|c|c|c|c|c|c|c|c|c|c|c|c|c|c|c|c|c|c|c|c|c|}
\hline & \multicolumn{4}{|c|}{1794} & \multicolumn{3}{|c|}{1794} & \multicolumn{5}{|c|}{1794} & \multicolumn{3}{|c|}{1794} & \multicolumn{5}{|c|}{1794} & \multicolumn{3}{|c|}{1794} \\
\hline & 1 & & 21 & & 1 & & 21 & & 1 & & 20 & & 1 & & 19 & & 1 & & 19 & & 1 & & 18 \\
\hline & 2 & & 22 & & 2 & & 22 & & 2 & $\mathrm{M}$ & 21 & & 2 & $\mathrm{~J}$ & 20 & & 2 & $\mathrm{~J}$ & 20 & & 2 & & 19 \\
\hline & 3 & $\mathrm{M}$ & 23 & & 3 & A & 23 & & 3 & & 22 & & 3 & & 21 & & 3 & & 21 & & 3 & A & 20 \\
\hline & 4 & A & 24 & & 4 & $\mathrm{P}$ & 24 & & 4 & A & 23 & & 4 & $\mathrm{u}$ & 22 & & 4 & $\mathrm{u}$ & 22 & & 4 & $\mathrm{U}$ & 21 \\
\hline & 5 & $\mathrm{R}$ & 25 & & 5 & $\mathrm{R}$ & 25 & & 5 & & 24 & & 5 & & 23 & & 5 & & 23 & $\mathrm{~F}$ & 5 & G & 22 \\
\hline & 6 & $\mathrm{C}$ & 26 & & 6 & I & 26 & & 6 & $\mathrm{Y}$ & 25 & & 6 & $\mathrm{~N}$ & 24 & $\mathrm{~T}$ & 6 & $\mathrm{~L}$ & 24 & & 6 & $\mathrm{U}$ & 23 \\
\hline \multirow[t]{2}{*}{ G } & 7 & $\mathrm{H}$ & 27 & & 7 & $\mathrm{~L}$ & 27 & $\mathrm{P}$ & 7 & & 26 & M & 7 & & 25 & & 7 & & 25 & $\mathrm{R}$ & 7 & S & 24 \\
\hline & 8 & & 28 & $\mathrm{~F}$ & 8 & & 28 & & 8 & & 27 & & 8 & $\mathrm{E}$ & 26 & $\mathrm{H}$ & 8 & $\mathrm{Y}$ & 26 & & 8 & $\mathrm{~T}$ & 25 \\
\hline \multirow[t]{2}{*}{$\mathrm{E}$} & 9 & & 29 & & 9 & & 29 & $\mathrm{R}$ & 9 & & 28 & $\mathrm{E}$ & 9 & & 27 & & 9 & & 27 & $\mathrm{U}$ & 9 & & 26 \\
\hline & 10 & & 30 & $\mathrm{~L}$ & 10 & & 30 & & 10 & & 29 & & 10 & & 28 & $\mathrm{E}$ & 10 & & 28 & & 10 & & 27 \\
\hline \multirow[t]{2}{*}{$\mathrm{R}$} & 11 & & 1 & & 11 & & 31 & A & 11 & & 30 & $\mathrm{~S}$ & 11 & & 29 & & 11 & & 29 & $\mathrm{C}$ & 11 & & 28 \\
\hline & 12 & & 2 & $\mathrm{O}$ & 12 & & 1 & & 12 & & 31 & & 12 & & 30 & $\mathrm{R}$ & 12 & & 30 & & 12 & & 29 \\
\hline
\end{tabular}




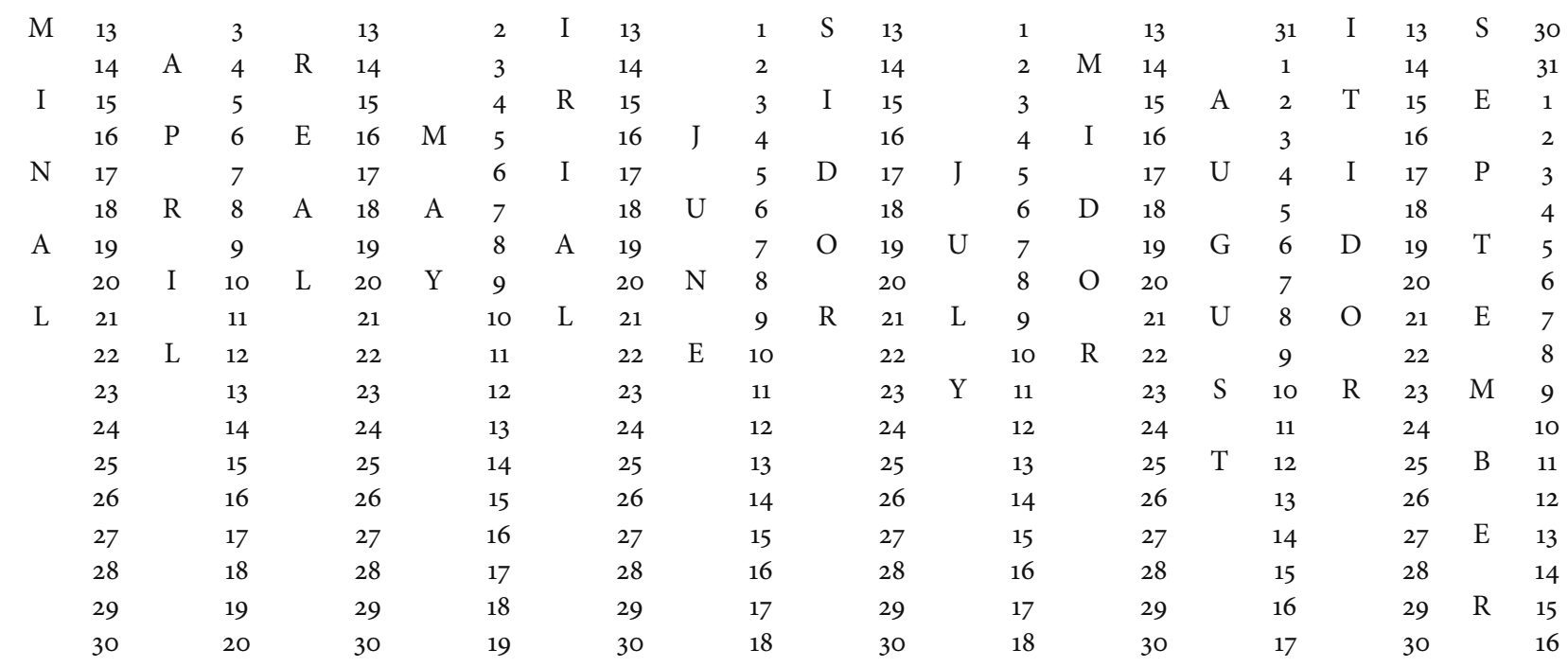

Jours Complémentaires Sans-culottides 
Jonathan Smyth - 9781526103802

Downloaded from manchesterhive.com at 04/26/2023 09:09:20AM 